\title{
Application of Bio-inspired Formation Flight Control to Two Fixed Wing Airplanes
}

\author{
Yoshinobu Inada*, Yuta Hayashi, and Yohei Koyama \\ Department of Aeronautics and Astronautics, School of Engineering, \\ Tokai University, Japan
}

\begin{abstract}
Formation flight control is an effective method for small unmanned air vehicles (UAVs) to improve the limited performance of an individual UAV in a powerful aggregated system as a group. In this study, formation flight control with two fixed wing airplanes as examples of UAVs were investigated based on the collective motion of organisms where simple local interactions, such as, attraction, repulsion, and parallel orientation, form an orderly motion. A control system for the aforementioned interactions was developed using a microcomputer, motion sensor, direction sensor, GPS, and communication devices and was implemented on two fixed wing type airplanes. Flight tests were conducted for the attraction-repulsion and the parallel orientation controls, and the cooperated flight of two fixed wing airplanes was successfully accomplished, indicating the feasibility of the bio-inspired formation flight control.
\end{abstract}

Index Terms - Collective motion, Formation flight control, Fixed wing airplane

\section{INTRODUCTION}

Fish and birds are known to create large groups, as shown in Fig. 1. This collective motion is thought to have several biological benefits, like an efficient search of predators or food by monitoring with many eyes or other sensory organs. Insects also create large groups for the efficient transportation of a subject with many collaborative carriers, like ants or honeybee swarms [1, 2].

These biological advantages are also beneficial for artificial systems, like information gathering or rescue systems, which require the features of efficient

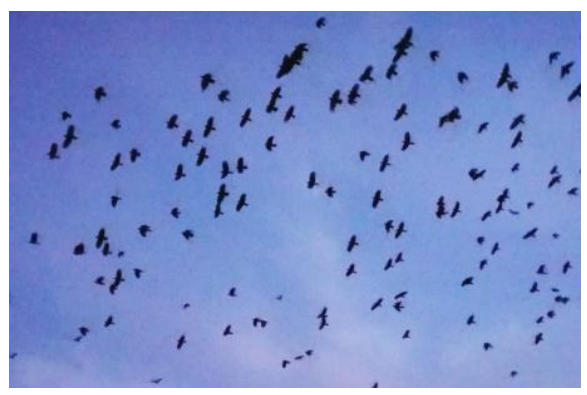

Fig. 1 Bird flock (taken by the author) monitoring or transportation. The formation flight of multiple unmanned air vehicles (UAVs) is a suitable example of these artificial systems. The use of many UAVs can realize efficient aerial surveillance or transportation systems [3-6]. Information display is another effective application; a famous example is the "Shooting Star" of Intel [7]. A large number of quadcopters with LEDs were operated cooperatively to create large characters and figures, etc., in the sky as a light show. The application as a military system is also spreading worldwide as a strategy to reduce the loss of human life and expensive equipment in battlefields [8].

These artificial collective motions are predominantly based on top-down control, in which a small number of strong leaders or host computers control the entire system. This type of control can realize a highly ordered system with a rigid hierarchy, but, inevitably, it has a weakness of durability because a malfunction of leaders or host computers will cause a breakdown of the whole system. Collective systems in nature avoid this problem by eliminating leaders in the group and forming a group with uniform members, as shown in many types of fish school or bird flock [9-12].

Previous studies have clarified that natural collective motion is achieved by a simple local interaction consisting of three behavioral rules, i.e., "attraction", "repulsion", and "parallel orientation", as seen in fish schools [9-11], bird flocks [12], mammal herds [13], and bio-inspired theoretical models [14-19]. Attraction and repulsion adjust the distance between individuals in a group so that the individual approaches the neighbor when it is too distant and retreats when it is too close. Meanwhile, parallel orientation orients the individual in the same direction as its neighbors.

This simplicity considerably contributes to decreasing the cost of conducting collective motion because the load for each individual that forms a group depends only on local interaction; thus, it is almost constant, even when the group size increases. This is not the case for the topdown control currently used in many artificial collective systems, where the load tends to be focused on the leaders or host computers and, thus, the system may easily break down due to an overload as the group size increases.

The novelty of this study is to apply the principle of collective motion in nature to artificial systems, with the aim of realizing the formation flight of multiple small UAVs. Small UAVs generally have a size and weight 


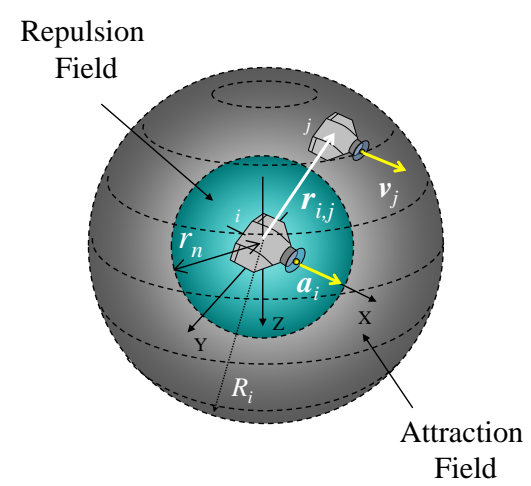

Fig. 2 Interaction field

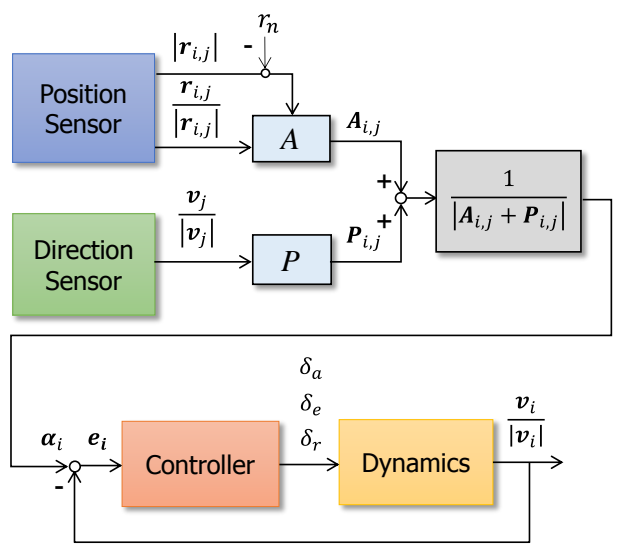

Fig. 3 Control model for the moving direction

that can be handled by one person, e.g., a wingspan < $1500 \mathrm{~mm}$ and weight $<2.0 \mathrm{~kg}$ [20] in the case of an airplane. This is advantageous over larger UAVs regarding purchasing and handling costs, but is disadvantageous regarding payload, durability, and operability. The use of multiple UAVs can solve these problems through the integration of a limited payload of small UAVs, by continuing their missions should some become lost, as well as by combining different devices on different UAVs for uses of various purposes.

Based on the above background information, we studied the formation flight control of multiple UAVs with reference to collective motions in nature. We first attempted to accomplish the formation flight control of two fixed wing airplanes. Since the control is based on the local interaction between members, the basic schemes for multiple and two aircraft are the same. Hence, the proposed methods for the two airplanes may be effective for multiple aircraft.

Details of the methods and results are explained in the following sections.

\section{METHODS}

\section{A. Fundamental Model}

For the formation flight control in this study, an interaction field was set around each airplane, as shown in Fig. 2. This field consisted of two subfields; an attraction field and a repulsion field. The motion of each individual was determined according to the position of its neighbors in the interaction field.

For the attraction-repulsion control, the individual approached a neighbor when the neighbor was in the attraction field and retreated when the neighbor was in the repulsion field. The advancing direction of the individual was then determined by the following attraction-repulsion vector $\boldsymbol{A}_{i, j}$ :

$$
\boldsymbol{A}_{i, j}=A\left(\left|\boldsymbol{r}_{i, j}\right|-r_{n}\right) \frac{\boldsymbol{r}_{i, j}}{\left|\boldsymbol{r}_{i, j}\right|}
$$

where $\boldsymbol{r}_{i, j}$ is the position vector of the neighbor ( $j$-th individual) from the individual at the center of the interaction field ( $i$-th individual); $r_{n}$ is the neutral distance where the attraction and the repulsion are balanced; $A$ is the attraction-repulsion gain. All vectors and the distances in this study were normalized by the body length of the individual so that the scale effect did not affect the control.

For the parallel orientation control, the $i$-th individual oriented parallel with the $j$-th individual in the interaction field. The advancing direction of the $i$-th individual was then determined by the following parallel orientation vector $\boldsymbol{P}_{i, j}$ :

$$
\boldsymbol{P}_{i, j}=P \frac{\boldsymbol{v}_{j}}{\left|\boldsymbol{v}_{j}\right|}
$$

where $\boldsymbol{v}_{j}$ is the velocity vector of the $j$-th individual and $P$ is the parallel orientation gain.

Finally, the direction of movement of the $i$-th individual was given by the following vector $\boldsymbol{a}_{i}$ by addition of the two vectors $\boldsymbol{A}_{i, j}$ and $\boldsymbol{P}_{i, j}$ as follows:

$$
\boldsymbol{a}_{i}=\frac{\boldsymbol{A}_{i, j}+\boldsymbol{P}_{i, j}}{\left|\boldsymbol{A}_{i, j}+\boldsymbol{P}_{i, j}\right|}
$$

When multiple neighbors existed in the interaction field, the averaged $\boldsymbol{a}_{i}$ for all neighbors in the interaction field was used as the advancing direction of the $i$-th individual.

In the case of the airplane, the moving direction was controlled by three control surfaces; aileron, elevator, and rudder. Their angles, $\delta_{a}, \delta_{e}, \delta_{r}$, respectively, were determined based on the direction difference between the current flight direction of the $i$-th individual and the direction of $\boldsymbol{a}_{i}$. The block diagram of this moving direction control is shown in Fig. 3.

The flight speed was controlled so that the $i$-th individual could maintain an adequate distance (the neutral distance) to the $j$-th individual in such a way that the speed increased or decreased when the $i$-th individual delayed or advanced from the $j$-th individual, respectively. The attraction-repulsion vector $\boldsymbol{A}_{i, j}$ was then used for this speed control. The throttle was determined in proportion to the forward or backward component of $\boldsymbol{A}_{i, j}$ as follows: 


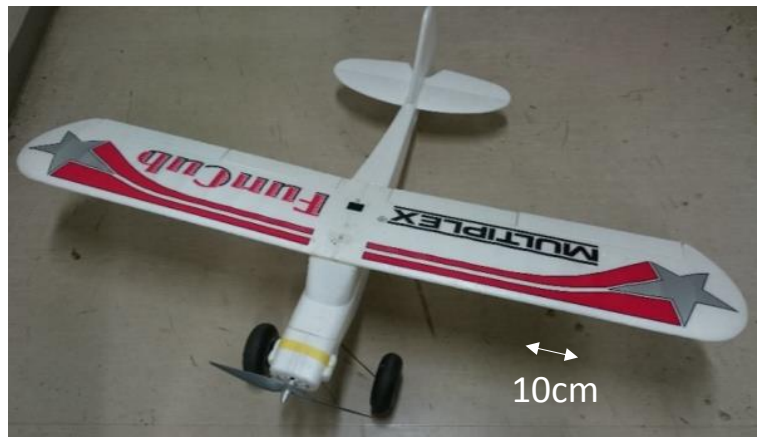

Fig. 4 Fixed wing airplane (FUN CUB)

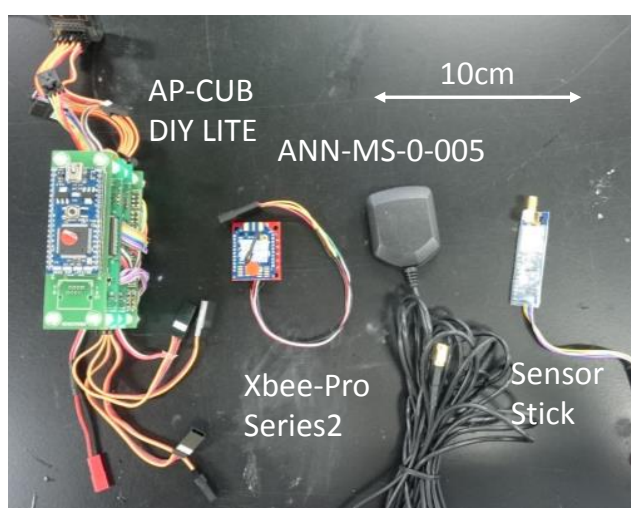

Fig. 5 Control devices

$$
\delta_{t}=\delta_{t . s}+T \boldsymbol{A}_{i, j} \cdot \boldsymbol{k}_{x}
$$

where $\delta_{t}$ and $\delta_{t . s}$ are the throttle and the throttle for steady flight, respectively; $T$ is the throttle gain; $\boldsymbol{k}_{x}$ is the normal vector orienting forward for the $i$-th individual.

In summary, the control parameters were the angles of the three control surfaces $\delta_{a}, \delta_{e}, \delta_{r}$, and the throttle $\delta_{t}$.

\section{B. The Practical Application of the Fundamental}

\section{Model to the Experiment}

Since this was the first trial of the fundamental model, the experimental condition was simplified as follows: the flightpaths of two airplanes were given on horizontal planes. The height difference of the two horizontal planes was maintained more than $5 \mathrm{~m}$ to avoid collisions. All vectors and flightpaths were determined on these horizontal planes.

The position vector $\boldsymbol{r}_{i, j}$ was calculated using the position information taken by the GPS sensor of each individual. The position information of the $j$-th individual was transmitted to the $i$-th individual using communication devices, then $\boldsymbol{r}_{i, j}$ was calculated on the $i$ th individual. The neutral distance was $30 \times$ body length

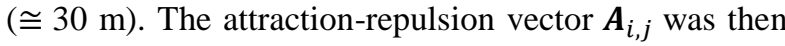
calculated using Eq. (1).

The moving direction of the $j$-th individual $\left(\boldsymbol{v}_{j} /\left|\boldsymbol{v}_{j}\right|\right)$ was taken by the magnetic direction sensor on the $j$-th individual and transmitted to the $i$-th individual, similarly using the communication devices. The parallel orientation vector $\boldsymbol{P}_{i, j}$ was then calculated using Eq. (2).
Next, the moving direction vector $\boldsymbol{a}_{i}$ was calculated using Eq. (3).

The moving direction of each airplane was controlled only by the rudder. The aileron was not used because the yaw made by the rudder induced the roll necessary for the turn by the dihedral angle effect of the wing. The elevator was not used either because the flightpath was almost horizontal during the flight test. The throttle was kept constant because the flight speed was almost constant in the horizontal flight. Hence, the only control parameter was the rudder angle $\delta_{r}$ in the flight test. In case of emergency, for example, an airplane lost height and almost crashed, the human pilot controlled the airplane using all control parameters.

The attraction-repulsion and the parallel orientation controls were tested separately in the flight test. The two gains $A$ and $P$ were $A=1.0$ and $P=0.0$ for the attractionrepulsion control test, and $A=0.0$ and $P=1.0$ for the parallel orientation test. The positive value of the gains could be given arbitrarily because they were cancelled in Eq. (3).

\section{Experimental Equipment}

The fixed wing airplane used in the experiment was FUN CUB (Multiplex Modellsport Ltd., Germany) made of styrene foam with a $1400 \mathrm{~mm}$ wingspan and $980 \mathrm{~mm}$ body length, as shown in Fig. 4. Its weight was $1130 \mathrm{~g}$ and the wing had a $2^{\circ}$ dihedral angle. Two of the same airplanes were used for the formation flight.

The control system for each airplane consisted of a mainboard, a sensor stick, a GPS antenna, and a communication device. The information of each device (manufacturing company, type, and performance) is shown in Table 1 and the appearance in Fig. 5. Although

Table 1 Control device list

\begin{tabular}{|c|c|c|}
\hline Device & Company & Type \& Performance \\
\hline Mainboard & $\begin{array}{l}\text { XENOCROSS } \\
\text { Co., Ltd. }\end{array}$ & $\begin{array}{l}\text { AP-CUB DIY LITE } \\
\text { CPU: H8S/2638, } 20 \mathrm{MHz}\end{array}$ \\
\hline Sensor stick & CSG Shop & $\begin{array}{l}\text { Acceleration: BMA180 } \\
\text { X, Y, Z: } \pm 4 \text { g, } 50 \mathrm{~Hz} \\
\text { Gyro: ITG3200 } \\
\text { X, Y, Z: } \pm 2000 \% \text { s, } 50 \mathrm{~Hz} \\
\text { Magnetic direction: } \\
\text { HMC5883L } \\
\text { X, Y, Z: } \pm 8 \text { G, } 1 \mathrm{~Hz} \\
\text { Air pressure: BMP085 } \\
300-1100 \mathrm{hPa}, 10 \mathrm{~Hz} \\
\text { GPS: ublox NEO-M8N } \\
2 \mathrm{~Hz}\end{array}$ \\
\hline GPS antenna & U-blox Inc. & ANN-MS-0-005 \\
\hline $\begin{array}{l}\text { Communication } \\
\text { device }\end{array}$ & $\begin{array}{l}\text { Digi } \\
\text { International } \\
\text { Inc, }\end{array}$ & $\begin{array}{l}\text { Xbee-Pro Series } 2 \\
\text { Comm. Speed: } 57600 \text { bps, } \\
\text { Max. Comm. Distance } \\
\text { (outdoor): } 1500 \mathrm{~m}, 5 \mathrm{~Hz}\end{array}$ \\
\hline
\end{tabular}




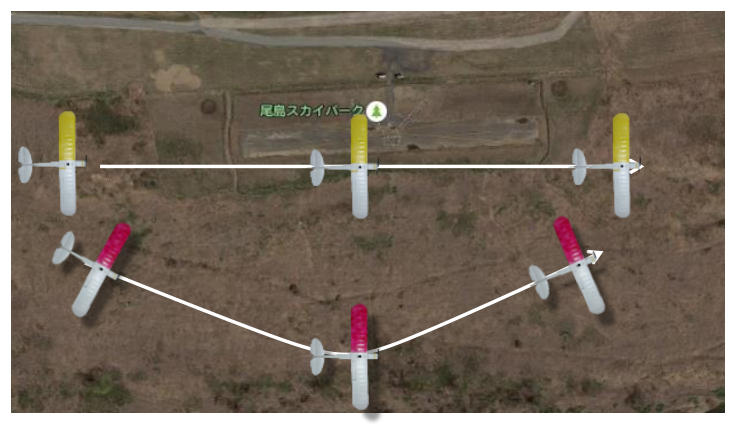

(a) Approach-repulsion

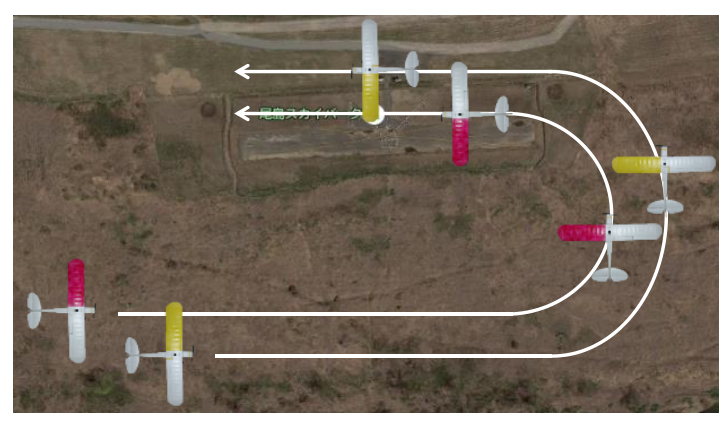

(b) Parallel orientation

Fig. 6 Flight test (yellow: HCA, red: ACA)

the airspeed sensor was not included in the system, all the necessary information for the control was taken from the GPS and the magnetic direction sensor.

\section{Flight Test}

Two different flight tests, the attraction-repulsion control test and the parallel orientation control test, were conducted using two airplanes. One airplane was controlled by a human pilot to be used as the stable target for the other airplane to conduct each control. The second plane was controlled automatically by the attraction-repulsion or the parallel orientation control. The automatically controlled airplane (ACA) did not receive any command from the human-controlled airplane (HCA). It only received the position and the direction information from the HCA and decided its motion independently. Therefore, the "leader" and "follower" relationship between the two airplanes did not exist in the formation flight in this study.

For the attraction-repulsion control, the two airplanes were initially close to each other; the distance between them being smaller than the neutral distance $r_{n}$. The HCA was controlled to fly over the test area. The ACA was controlled by the attraction-repulsion control, assuming it would initially move away from the HCA before approaching it when the distance between them was larger than $r_{n}$, as shown in Fig. 6(a).

For the parallel orientation control, the two airplanes were initially oriented in the same direction, the HCA was controlled to move along a U-shaped trajectory,

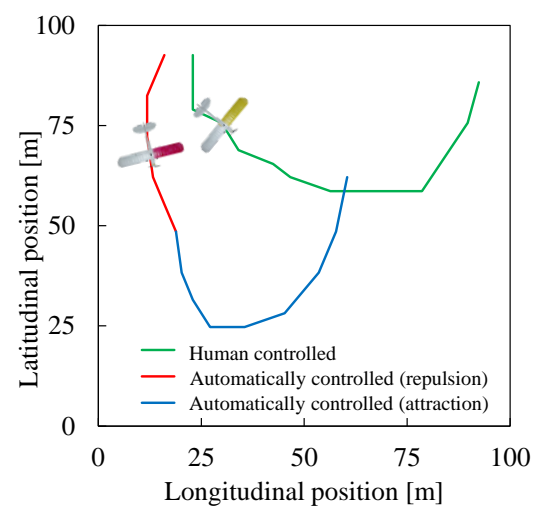

(a) Trajectory

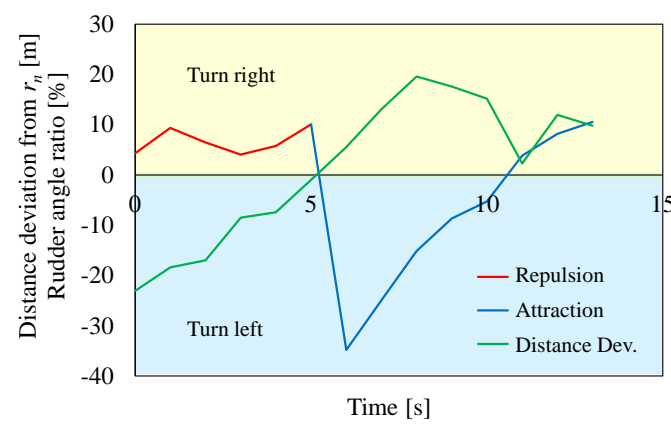

(b) Rudder angle and distance deviation

Fig. 7 Result of flight test for the attractionrepulsion control

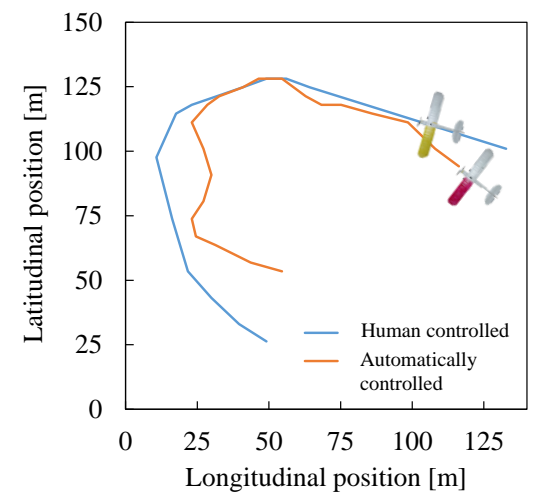

(a) Trajectory

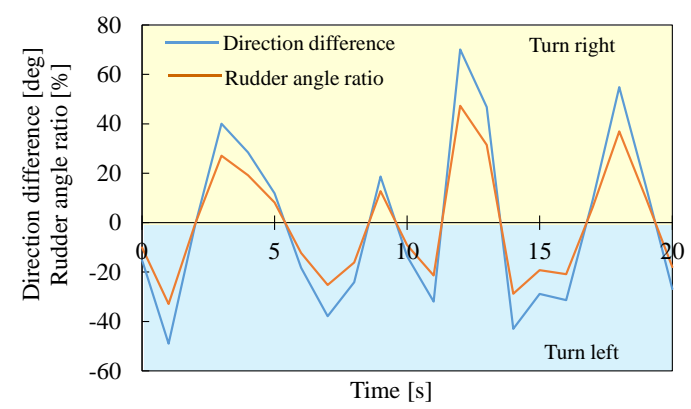

(b) Rudder angle and direction difference

Fig. 8 Result of flight test for the parallel orientation control 
while the ACA was controlled by the parallel orientation control, assuming it would maintain its orientation parallel with the HCA, as shown in Fig. 6(b).

\section{RESULTS AND DISCUSSION}

\section{A. Attraction-Repulsion Control Test}

During the flight test of the attraction-repulsion control, the ACA initially tried to move away from the HCA, as shown in Fig. 7(a) (red line). Throughout this motion, the deviation of the distance between the two airplanes from the neutral distance $r_{n}$ was negative, i.e., the distance between the two airplanes was smaller than $r_{n}$, as shown in Fig. 7(b) (green line), and the rudder angle ratio of the ACA, determined as the ratio of the rudder angle to the maximum rudder angle $\left(= \pm 20^{\circ}\right)$, was positive, as shown in Fig. 7(b) (red line). This indicates that the ACA was controlled to turn right, to move away from the HCA, thus showing that the repulsion control was adequately operated by the ACA.

The ACA tried to approach the HCA when they were apart, as shown in Fig. 7(a) (blue line). During this period, the distance deviation from $r_{n}$ became positive, showing the distance between the two airplanes was larger than $r_{n}$, as shown in Fig. 7(b) (green line) and the ladder angle ratio became negative, as shown in Fig. 7(b) (blue line). This indicates that the ACA was controlled to turn left to approach the HCA, thus, the approach control was adequately operated by the ACA.

Although the flightpaths of the two airplanes crossed at one point, as shown in Fig. 7(a), they did not pass this point at the same time and their heights differed by more than $5 \mathrm{~m}$. Hence, the two airplanes were safely apart from each other at this point. Height loss of the ACA did not occur during the flight test. Therefore, there was no human intervention in the ACA during the flight test.

Two or three flight tests were conducted, including the one mentioned above, and similar flightpaths and rudder motions were taken in all cases (see Appendix). Therefore, these results show that both the attraction and repulsion control were successfully achieved in the flight test.

\section{B. Parallel Orientation Control Test}

In the flight test of parallel orientation control, the recording of flight was started shortly before the two airplanes began turning. Both the HCA and ACA maintained orientation similar directions during the flight, as shown in Fig. 8(a). The rudder angle ratio of the ACA was proportional to the direction difference between the two airplanes, as shown in Fig. 8(b), indicating that the ACA operated the rudder to decrease the direction difference.

The fluctuation in the graph may be caused by the delay of the airplane motion, as the airplane could not stop turning immediately as the rudder angle became zero, resulting in the overshooting of the direction difference. There was little delay in the control system because the rudder followed the change of the direction difference with almost no delay. The first fluctuation $(0$ $-6 \mathrm{~s})$ and the second fluctuation $(6-12 \mathrm{~s})$ showed a convergence. The third large fluctuation was caused by initiation of a left turn of the HCA $(12-15 \mathrm{~s})$, while it converged in the fourth fluctuation $(15-20 \mathrm{~s})$. Thus, these results indicate that the parallel orientation control was adequately operated on the ACA.

Three flight tests were conducted, including that mentioned above, and similar flightpaths and rudder motions were taken in all cases. Therefore, these results show that the parallel orientation control was successfully achieved in the flight test.

\section{Effect of the Control System's Performance on the Flight Test}

The performance of the control system, shown in Table 1, was sufficient for conducting either the attraction-repulsion control or the parallel orientation control in this study. Among the devices, the GPS generally has a position error of approximately 5 or 10 $\mathrm{m}$, however, the serious problem of an airplane collision could be avoided by appointing a larger neutral distance $r_{n}(\cong 30 \mathrm{~m})$. Although fluctuations were observed in the direction difference, as shown in Fig. 8(b), the average value of the direction difference during the flight test was $-1.26^{\circ}$, indicating that the direction error was sufficiently small. There were no serious disruptions caused by the communication between the two airplanes during the flight test.

However, insufficiency of performance occurred when both the attraction-repulsion and the parallel orientation controls were executed simultaneously. The control system failed many times due to being overloaded. This problem may also occur in a formation flight with multiple (> 2) airplanes. Hence, the system requires improvements to be made in future research.

\section{CONCLUSION}

This study investigated the formation flight control of two fixed wing airplanes based on natural collective motion control. Three kinds of local interactions, i.e., "attraction", "repulsion", and "parallel orientation", the basic interactions for achieving the collective motion of organisms in nature, were applied to automatically controlled airplanes equipped with microcomputer, motion and direction sensors, GPS, and communication devices. Flight tests for the attraction-repulsion control and the parallel orientation control were conducted and the results indicate that the attraction-repulsion control was adequately carried out by programming the airplane to approach or retreat from its neighbor according to the distance between them, measured by GPS. The parallel orientation control was also adequately performed by maintaining the orientation of both the airplanes to be almost parallel with each other according to the moving direction measured by the magnetic direction sensors.

This study confirms that the two controls, attractionrepulsion and parallel orientation, were successfully realized using microcomputer, mini-size sensors, and communication devices on each airplane. Although only two airplanes were used in the test, a similar interaction 
mechanism can be applied to multiple airplane cases. Thus, this result provides a positive prospect for the application of bio-inspired collective motion control to the formation flight of multiple aircraft.

However, the simultaneous execution of both controls and the formation flight control of multiple airplanes did not succeed in the flight test due to the insufficiency of the control system. Improvements of the devices and programs are required to successfully achieve bioinspired formation flight control using three local interactions with multiple airplanes.

\section{ACKNOWLEDGEMENTS}

The authors express special thanks to Dr. Mitsutake from XENOCROSS. Co., Ltd. for providing information on the control devices used herein.

\section{REFERENCES}

[1] McFarland, D., "Oxford Companion to Animal Behavior", Oxford University Press (1987).

[2] Marshall, N. B., "The Life of Fishes", Universe Books, New York (1970).

[3] Nigam, N., Bieniawski, S., Kroo, I., Vian, J., "Control of Multiple UAVs for Persistent Surveillance: Algorithm and Flight Test Results", IEEE Transactions on Control Systems Technology, Vol. 20, Issue 5 (2012), pp.1236-1251.

[4] Scherer, J., Rinner, B., "Persistent multi-UAV surveillance with energy and communication constraints", 2016 IEEE International Conference on Automation Science and Engineering (CASE), 21-25 Aug. (2016).

[5] Shirania, B., Najafib, M., Izadia, I., "Cooperative load transportation using multiple UAVs", Aerosp. Sci. Technol., Vol. 84 (2019), pp.158-169.

[6] Thapa, S., Bai, H., Acosta, J. A., "Cooperative Aerial Load Transport with Force Control”, IFACPapersOnLine Vol. 51, Issue 12 (2018), pp. 38-43.

[7] Um, J. S., "Drones as Cyber-Physical Systems", Springer Singapore (2019).

[8] Callam, A., "Drone Wars: Armed Unmanned Aerial Vehicles", International Affairs Review (http://www. iar-gwu.org/node/144), Vol. XVIII, No. 3: Winter (2010).

[9] Aoki, I., "An Analysis of the Schooling Behavior of Fish: Internal Organization and Communication Process", Bull. Ocean. Res. Inst. Vol. 12 (1980), pp.1-64.

[10] Inagaki, T., Samamoto, W., Aoki, I., "Study on the Schooling Behavior of Fish-IV, some discussions concerning continuity of swimming speed and response time of individuals", Bul. Jpn. Soc, Sci, Fish. Vol. 42 (1976), pp.1083-1091.

[11] Grunbaum, D., "Schooling as a Strategy for taxis in a Noisy Environment”, Evol. Ecol. Vol. 12 (1998), pp.503-522.

[12] Heppner, F., "Three-dimensional Structure and Dynamics of Bird Flocks", Parrish, J. K. and Hamner, W. M. ed., Animal Groups in Three
Dimensions, Cambridge University Press (1997), pp.68-89.

[13] Gueron, S., Levin, S. A., Rubenstein, D. I., "The Dynamics of Herds, from Individual to Aggregations", J. theor. Biol. Vol. 182 (1996), pp.85-98.

[14] Huth, A., and Wissel, C., "The Simulation of the Movement of Fish Schools", J. theor. Biol. Vol. 156 (1992), pp.365-385.

[15] Inada, Y. and Kawachi, K., "Order and Flexibility in the Motion of Fish Schools", J. theor. Biol. Vol. 214 (2002), pp.371-387.

[16] Matuda, K., and Sannomiya, N., "Computer Simulation of Fish Behavior in Relation to Fishing Gear", Bul. Jpn. Soc, Sci, Fish. Vol. 46 (1985), pp.689-697.

[17] Niwa, H.-S., "Self-organizing Dynamic Model of Fish School", J. theor. Biol. Vol. 171 (1994), pp.123-136.

[18] Inada, Y., and Takanobu, H., "Dependency of Collective Motion Control of Air Vehicles on Interaction Parameters", Theoretical and Applied Mechanics Japan, Vol. 58 (2010), pp.185-195.

[19] Warburton, K., and Lazarus, J., "Tendencydistance Models of Social Cohesion in Animal Groups", J. theor. Biol. Vol. 150 (1991), pp.478488.

[20] Fahlstrom, P. G., and Gleason, T., "Introduction to UAV Systems 4th Edition", John Wiley \& Sons, Ltd. (2012). 


\section{APPENDIX}

Results of other flight tests for the attraction-repulsion and parallel orientation control

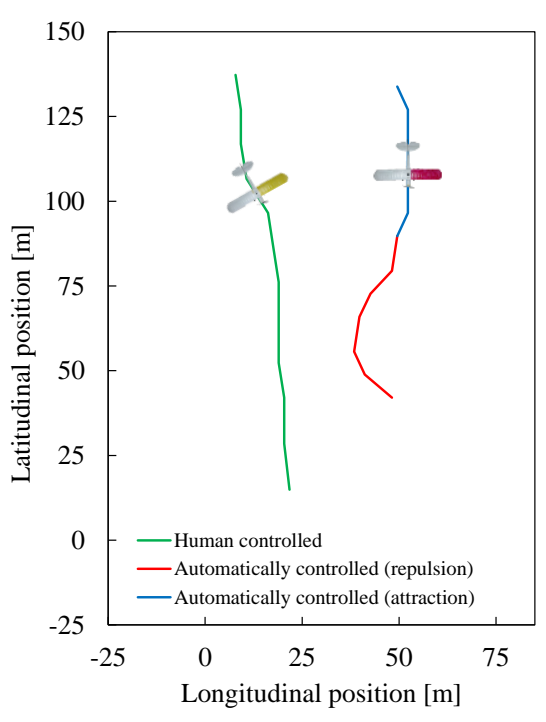

(a) Trajectory (Case 2)

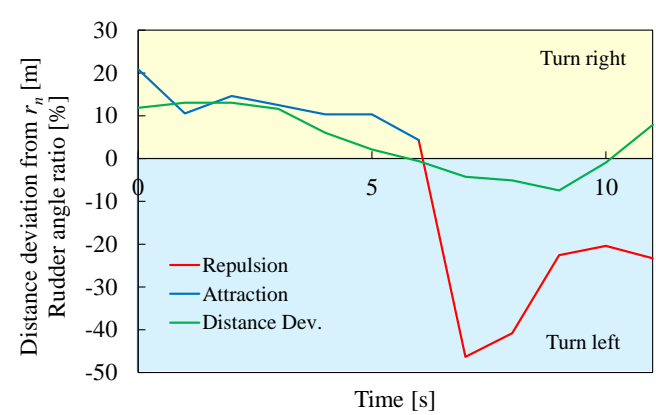

(b) Rudder angle and distance deviation (Case 2)

Fig. A-1 Result of flight test for the attractionrepulsion control

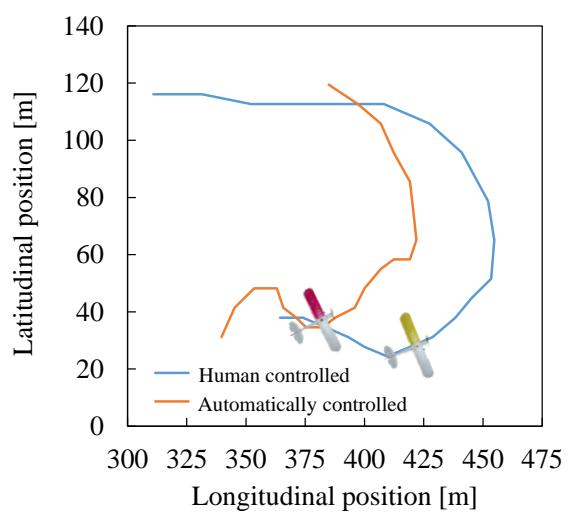

(a) Trajectory (Case 2)

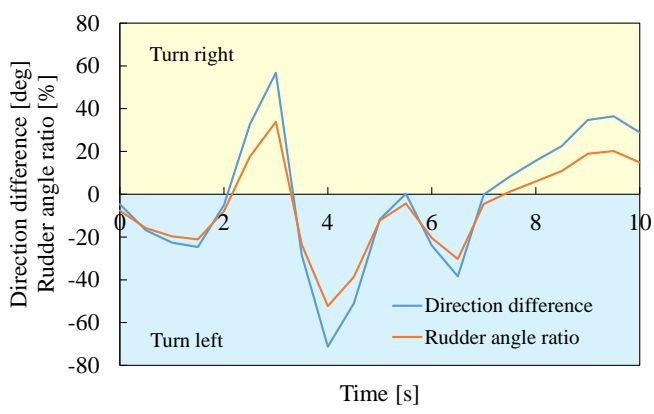

(b) Rudder angle and direction difference (Case 2)
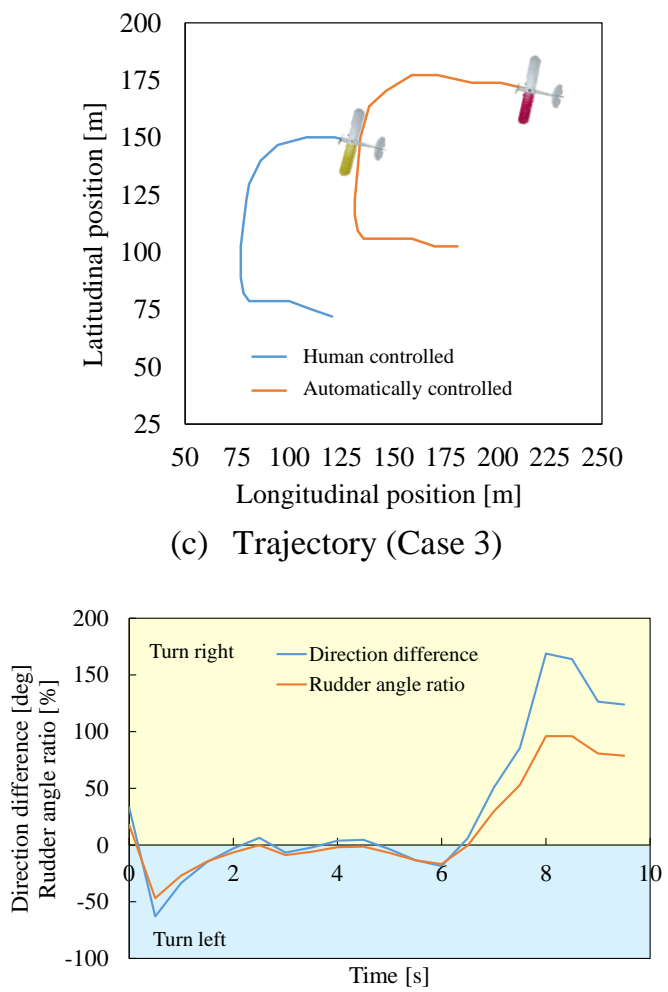

(d) Rudder angle and direction difference (Case 3)

Fig. A-2 Result of flight tests for the parallel orientation control 